\title{
El amor en educación física: una perspectiva filosófica basada en Max Scheler y José Ortega y Gasset \\ Love in physical education: a philosophical perspective based on Max Scheler and José Ortega y Gasset \\ *Felipe Nicolás Mujica Johnson, **Nelly Del Carmen Orellana Arduiz \\ *Universidad Católica de Temuco (Chile), **Universidad de Playa Ancha (Chile)
}

Resumen: La actividad filosófica se dirige principalmente al mundo de las ideas, o sea, trata sobre el pensamiento y la meditación de los temas más profundos de la existencia humana y no humana. Por otra parte, la actividad pedagógica se dirige principalmente a fines más prácticos, aunque no menos importantes, y aprovecha los saberes desvelados por los diferentes referentes de la filosofía. No obstante, se ha señalado que por diferentes motivos la educación se ha alejado mucho del mundo más profundo de las ideas, lo cual puede derivar en una actividad pedagógica muy superficial. Con este ensayo se pretende acercar la filosofía a la educación y, en concreto, a la educación física. En primer lugar, se tuvo como objetivo analizar el significado de amor que proponen dos filósofos asociados a la escuela fenomenológica, Max Scheler y José Ortega y Gasset. Posteriormente, aquel significado se asoció a la formación ética en la educación física escolar. El análisis refleja que ambos coinciden en que el amor es un sentimiento objetivo y un acto intencional dirigido a conservar adecuadamente lo que es valorado positivamente. También reconocen que el amor es anterior a las emociones, los pensamientos y los deseos, así como que su dirección va desde lo superior a lo inferior. Este conocimiento puede ser aplicado en la educación física de diferentes formas, destacando la conciencia sobre la relación de las emociones con los valores éticos, como la solidaridad, el respeto a los derechos humanos, la tolerancia y la humildad.

Palabras clave: emociones, ética, educación física, derechos humanos, filosofía.

Abstract: Philosophical activity is directed primarily to the world of ideas, that is, it deals with thoughts and meditation on the deepest themes of human and non-human existence. On the other hand, the pedagogical activity is mainly directed to more practical purposes, although not less important, and takes advantage of the knowledge revealed by the different referents of philosophy. However, it has been pointed out that for different reasons education has strayed far from the deeper world of ideas, which can lead to very superficial pedagogical activity. This essay aims to bring philosophy closer to education and, specifically, to physical education. First, the objective was to analyze the meaning of love proposed by two philosophers associated with the phenomenological school, Max Scheler and José Ortega y Gasset. Later, that meaning was associated with ethical training in school physical education. The analysis reflects that both agree that love is an objective feeling and an intentional act aimed at adequately preserving what is positively valued. They also recognize that love precedes emotions, thoughts, and desires, as well as its direction goes from the top to the bottom. This knowledge can be applied in physical education in different ways, highlighting awareness on the relationship between emotions and ethical values, such as solidarity, respect for human rights, tolerance, and humility.

Key Words: emotions, ethics, physical education, human rights, philosophy.

\section{Introducción}

Este ensayo versará sobre el amor desde dos principales perspectivas filosóficas, las cuales serán asociadas a la actividad pedagógica y, en concreto, al ámbito de la educación física (EF). De esta forma, se pretende generar un vínculo entre algunas concepciones del amor y su potencial para la educación ética en el contexto escolar.

La actividad filosófica ha sido, y sigue siendo, muy importante para el desarrollo cultural en occidente, por ejemplo, al aportar parte de las bases teóricas en el ámbito del derecho, la política, la ciencia y la educación. Por ende, también de la EF (Cagigal, 1975, 1981a, 1981b, 1984, 1986, 1996; Devís, 1996; Kirk, 2010; Mujica, 2019, 2020a, 2020b). Sobre su significado, Scheler (1966), desde una perspectiva fenomenológica, indica que su esencia trata el «conocimiento del universo o sabiduría del universo» (p. 107). Dicha actividad suele ser reconocida por su trascendencia en la vida humana de las diversas naciones, pero lo que puede que no todo el mundo sepa, sobre todo las personas más noveles del ámbito educativo, es que tiene una enorme complejidad. Esto se aprecia en las diferentes corrientes de pen-

Fecha recepción: 07-04-20. Fecha de aceptación: 25-05-20 Felipe Nicolás Mujica Johnson fmujica@live.cl samiento que se han desarrollado, por ejemplo, el positivismo, el postpositivismo, la fenomenología, el existencialismo, el pragmatismo, el marxismo, el neomarxismo, el estructuralismo o el postestructuralismo (Ferrater, 1998). A su vez, también se refleja cuando ante un mismo concepto o ante una misma pregunta la filosofía nos puede proporcionar múltiples respuestas (Ferrater, 1994, 1995).

La dificultad que representa el estudio filosófico puede ser uno de los motivos que ha influido en su cada vez más dañada popularidad en el ámbito educativo en contraposición a la actividad científica. Por otra parte, se ha señalado como causante la diferente orientación intelectual de los filósofos y los educadores. Es decir, dado que, por una parte, «los filósofos están más interesados en el mundo de las ideas, para hacerlas encajar entre sí formando un sistema de pensamiento coherente; mientras que por otra, los educadores están volcados fundamentalmente en la realización de una práctica concreta» (Amilburu, 2014). Este problema que ha tenido la actividad filosófica se ha visto reflejado de diversas formas en la práctica pedagógica, lo cual se suma al desprecio de las humanidades en general, lo cual ha sido atribuido, en parte, a la mercantilización y deshumanización del proceso educativo (Mujica e Inostroza, 2020; Nussbaum, 2010, 2016). Precisamente, dicha lógica industrial que sigue muy implícita en el orden de los centros educativos, es a la 
que se atribuyen gran parte de las barreras para dignificar la EF y favorecer la innovación de sus metodologías de enseñanza en torno a sus variados contenidos didácticos (Kirk, 2010, 2017; Lawson, 2009, 2018, 2019; Siedentop, 2002). A pesar del distanciamiento, o la escasa interacción, que puede existir entre el mundo filosófico y el mundo pedagógico, diferentes especialistas realizan constantes esfuerzos para intentar aproximarlos (Almiburu, 2014; Mari, 2014). Y, por supuesto, también en el contexto de la EF (Cagigal, 1975, 1984, 1996; Castro, 2012; Mujica, 2020a; Oña, 2002; Perrino \& Vicente, 2018).

Este ensayo se enmarca esa línea de estudio que pretende acercar, o integrar, la actividad filosófica y educativa. En concreto, versará sobre el amor en el contexto específico de EF escolar. Para ello, se hará una aproximación al fenómeno de estudio desde la mirada de dos reconocidos filósofos, el alemán Max Scheler (1874, Múnich-1928, Fráncfort del Meno) y el español José Ortega y Gasset (1883, Madrid - 1955, Madrid). Se han seleccionado estos dos filósofos porque ambos compartieron una misma escuela o corriente filosófica, que es la fenomenología y lograron una mirada relativamente similar en torno al amor, por supuesto, con muchos matices. Otro punto muy importante del por qué se han escogido estos pensadores, responde a que se considera que ambos realizaron aportes significativos al tema de estudio y que pueden ser aplicados, provechosamente, en la labor pedagógica. En este sentido, desde una postura fenomenológica frente al tema y en contraposición a una postura positivista, se considera que la subjetividad de quien desarrolla este escrito ha sido fundamental para aproximarse al fenómeno estudiado (Fuster, 2019).

En términos generales, el amor ha sido dotado de múltiples significados, algunos de carácter vulgar o coloquial y otros de un carácter más riguroso o razonado. Esto es bien explicado por Ferrater (1994), quien manifestó que su término se ha empleado «para designar actividades $-\mathrm{o}$ el efecto de actividades- muy diversas; el amor es interpretado como inclinación, afecto, apetito, pasión, aspiración, etc. Otras veces se le ha considerado una cualidad, propiedad o relación» (p. 30). En el ámbito educativo el amor también suele utilizarse de diferentes formas y, evidentemente, sin mucha rigurosidad (Mujica \& Orellana, 2020), porque, como señaló Amilburu (2014), el contexto no lo exige. O sea, debido al pragmatismo que suele caracterizar el acto educativo, el amor, y muchos otros conceptos de relativo corte filosófico, suele ser dotado de un sentido común poco especificado. En el caso concreto de la EF, se ha reconocido que suele ser reducido a una emoción positiva para el bienestar subjetivo (Alonso, Marín, Yuste, Lavega \& Gea, 2019; FernándezGarcía \& Fernández-Río, 2019), la cual sería una posición contraria a la que se desarrollará en este ensayo.

Con base en lo mencionado anteriormente, este ensayo tiene por objetivo, en primer lugar, analizar el significado de amor que proponen dos filósofos asociados a la escuela fenomenológica y, posteriormente, asociarlo a la formación ética en la educación física escolar.

\section{Perspectiva filosófica del amor}

Las perspectivas filosóficas que se presentarán en este apartado tiene elementos en común, por ejemplo, tanto Scheler (2005) como Ortega y Gasset (2018), plantearon la existencia de un amor auténtico y que, a su vez, co-existen interpretaciones erradas en torno al significado y a las consecuencias del amor. Otra coincidencia de ambos filósofos, trata de su distinción del sentimiento del amor con las emociones, o sea, que es correcto considerar el amor como un sentimiento pero no como una emoción. Cabe destacar, que una emoción es entendida como una sensación intensa y de corta duración (Alonso, 2017; Rosas, 2011). En palabras del filósofo español, diríamos que la emoción es un sentimiento inerte y pasivo, debido a que «se <<está $>>$ triste o se $<<$ está $>>$ alegre, en pura pasividad. La alegría, por sí, no contiene actuación ninguna, aunque pueda llevar a ella. En cambio, amar algo no es simplemente $<<$ estar $>>$, sino actuar hacia lo amado» (Ortega y Gasset, 2018, p. 91). En este sentido, el filósofo de Múnich como el de Madrid se oponen a la interpretación de Spinoza sobre el amor, que lo reduce al estado y conocimiento en torno a la alegría (Ortega y Gasset, 2018; Scheler, 2005).

En los estudios de Ortega y Gasset (2018) sobre el amor, se aprecia la influencia alemana que tuvo del pensamiento scheleriano en sus ideas y, por otra parte, en los estudios de Scheler (2010) sobre el amor, queda muy clara la influencia que tuvo desde el pensamiento filosófico de San Agustín, Giordano Bruno y Blaise Pascal. Parte de esta relación, junto a otros datos muy significativos, la mencionó de esta forma Ferrater (1994) en su diccionario de filosofía:

Las ideas de Scheler - expresadas principalmente en su Ética, en naturaleza y formas de la simpatía y en sus estudios sobre $<<$ El pudor $>>\mathrm{y}<<$ ordo amoris $>>$ - tienen variedad de fuentes. Su pensamiento procede tanto de San Agustín como de Pascal, pero fundamentalmente se apoya en la teoría objetivista de los valores que él elaboró. Scheler negaba que el amor fuera una idea innata, que procediese exclusivamente de la experiencia o que fuera un impulso elemental (tal vez procedente de la líbido). Tanto para Scheler como para Brentano, el amor es un proceso intencional que trasciende hacia el ser amado, el cual es amado en razón de que es valorado alta y positivamente. No puede, pues, confundirse el amor con la compasión o la piedad. En tanto que acto intencional -o serie de actos- posee sus propias leyes, que no son psicológicas, sino axiológicas. El amor (como el odio) no es una tendencia de un sujeto psicológico, sino un acto personal que se manifiesta eligiendo o rechazando valores. Ni uno ni otro se definen, sino que se intuyen emotivamente y a priori. Así pues, para Scheler -como para San Agustín y Pascal- hay un ordo amoris (un orden en el amor), un ordre du coeur (un orden del corazón); en suma, el amor no es arbitrario, sino selectivo (pp. 35-36). A partir de sus posicionamientos, Scheler (2010) y Ortega y Gasset (2018), se posicionan a favor de que el amor es un sentimiento espiritual o metafísico, que funda otros sentimientos anímicos o psicológicos, deseos y razonamientos. O sea, se diferencian de otros pensadores que han reducido el amor al deseo (Santo Tomás de Aquino); al ámbito emocional (Spinoza); al ámbito pasional (Stendhal); y al conocimiento (Platón, Aristóteles y Buda). En cuanto a este último ámbito de corte intelectual, debido a la coincidencia del pensamiento griego con el hindú, Scheler (2010) lo llamó el pen- 


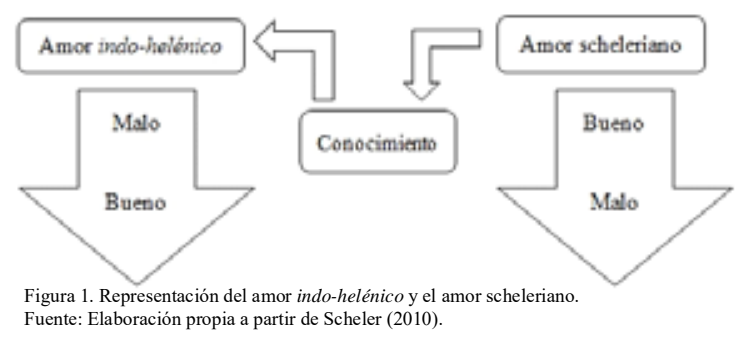

samiento indo-helénico del amor. La diferencia entre la idea indo-helénica del amor y la idea de scheleriana se expresa en la Figura 1. En dicha ilustración se puede observar dos diferencias en función de la dirección del amor y su relación con el conocimiento que es sostenida en las perspectivas scheleriana y orteguiana. En cuanto a la dirección, el amor indo-helénico se dirige de lo malo o inferior, a la bueno o superior, al contrario de la perspectiva scheleriana. Según el conocimiento, la concepción scheleriana señala que es producto del amor y, la perspectiva indo-helénica, plantea que el amor es producto del conocimiento. Sobre el error de reducir el amor a una consecuencia del conocer o saber, Ortega y Gasset (2018) señala lo siguiente:

El sumo error, desde el renacimiento hasta nuestros días, fue creer -con Descartes- que vivimos de nuestra conciencia, de aquella breve porción de nuestro ser que vemos claramente y en que nuestra voluntad opera. Decir que el hombre es racional y libre me parece una expresión muy próxima a ser falsa. Porque, en efecto, poseemos razón y libertad, pero ambas potencias forman sólo una tenue película que envuelve el volumen de nuestro ser, cuyo interior ni es racional ni es libre (p. 133).

Para los filósofos mencionados, el amor es un sentimiento metafísico y, por ende, objetivo, o sea, un acto intencional que pretende conservar lo que se reconoce como valioso. El amor antecede, es decir, es el trasfondo, de nuestros buenos sentimientos, pensamientos, deseos y actos humanos (Alves, Barea, Werneck, Grzibowski, Rodrigues \& Silva, 2018; Chaparro, 2014; Ortega y Gasset, 2018; Rodríguez, 2012; Scheler, 2001, 2005, 2008, 2010; Vendrell, 2015; Vlieghe \& Zamojski, 2019). Dado que el amor es conservación de lo valioso, se puede amar todo ser, objeto o actividad que se considere valiosa. En esta interpretación cobra sentido el amor al prójimo, a la ciencia, al arte, a la naturaleza o al deporte (Ortega y Gasset, 2018; Scheler, 2010). Scheler (2005) establece una categoría superior al amor que se dirige a la categoría de persona, dicho amor sería un amor moral, a diferencia, por ejemplo, del amor al arte. En sus palabras, sería que «el amor al valor de la persona, es decir, a la persona en cuanto realidad a través del valor de la persona, es el amor moral en sentido estricto» (Scheler, 2005, p. 231). Sobre la relación que tendría el amor con las emociones, ambos filósofos destacan que el amor se refleja en sus preferencias valóricas y, por ende, en sus procesos emocionales. Por ejemplo, quien ama a otra persona, se alegraría por lo bueno que le sucede y, en sentido contrario, se entristecería por lo malo que le suceda. Esta relación también se apreciaría en los actos intencionados de violencia para hacer daño a alguien, los cuales carecerían del sentimiento de amor, porque «cuanto más violento sea un acto psíquico, más bajo está en la jerarquía del alma» (Ortega y Gasset, 2018, p. 126). Cabe men- cionar que los y alcances en torno al amor de estos filósofos son mucho más complejos de los que se plantea en este ensayo (Chaparro, 2014; Rodríguez, 2012; Vendrell, 2015), a los cuales también se han presentado diferentes objeciones.

Para ir finalizando este apartado se hará referencia a un estudioso español del ámbito afectivo (Rojas, 1989), al psiquiatra español Enrique Rojas que le ha otorgado un espacio en su obra sobre la felicidad al pensamiento en torno al amor de ambos filósofos. Por cierto, destaca que, además de tener raíces en común, los fundamentos filosóficos que han aportado han sido muy valiosos para los profesionales de la psiquiatría. En el caso de Scheler, aportó una clasificación de los sentimientos que ha dado muchos frutos en el quehacer de esos profesionales de la salud. Por otra parte, Ortega y Gasset, por medio de su esclarecimiento del sentimiento del amor habría generado una positiva tensión en los profesionales dedicados a la psicología humana (Rojas, 1993). Se aprovechará la buena síntesis que realiza este psiquiatra español sobre el significado del amor de ambos intelectuales. En suma, el amor desde la mirada scheleriana sería «un proceso intencional que va más allá de lo que se ama; lo que se ama es valorado positivamente (...) no puede tenerse como algo arbitrario, que se va y que se viene; sino como un proceso emocional selectivo» (Rojas, 1993, p. 87). Por otro lado, desde la concepción orteguiana «el amor lleva a algo tan significativo que es capaz de entusiasmar y que puede comprometer toda la vida, teniendo, por ello, un fondo trágico, sobre todo si se acepta que la vida humana es en sí misma dramática» (Rojas, 1993, p. 89).

Cabe destacar que, desde una perspectiva filosófica y pedagógica, no se ha dicho la última palabra sobre lo que es o no es el amor, así como de su carácter objetivo y/o subjetivo, pues, si consideramos la perspectiva del filósofo danés Kierkegaard (2006), el origen del amor es un misterio muy difícil y hasta, posiblemente, imposible de resolver exhaustivamente por el ser humano. Por lo mismo, es necesario que se continúen realizando estudios sobre el tema y, sobre todo, en el marco de la pedagogía.

\section{Elamor en, y hacia, la EF}

Como bien han señalado diferentes especialistas de la $\mathrm{EF}$, esta asignatura no se debe enfocar en formar deportistas, atletas o personas exitosas en torno a los diferentes contenidos didácticos (Cagigal, 1996; Devís, 1996; Castejón, 2010). Otra debe ser su dirección, con un sentido mucho más humanista, y pedagógico, esta asignatura debe aprovechar todas sus diversas potencialidades para aportar al desarrollo, y aprendizaje, motor, y, a su vez, a la formación de buenas personas y, en consecuencia, buenos ciudadanos. O sea, una EF de buena calidad debe configurar su currículo y metodologías para contribuir a la educación integral del alumnado (Cagigal, 1975; 1984, 1986; Castejón, 2005; Hellison, 1995; Kirk, 2010; Lleixà, 2016; Mujica, 2019, 2020a; Velázquez \& Hernández, 2010). En concreto, ha de considerar el ámbito físico, motor, intelectual, afectivo y ético-moral. Precisamente, en aquel quehacer pedagógico es donde se pueden aprovechar los fundamentos filosóficos para iluminar las actuaciones educativas. Justamente, Vliegue y Zamojski (2019), basándose en el aporte de Scheler, manifiestan que el amor 
es fundamental para una adecuada educación escolar.

Los conocimientos que nos proporciona la perspectiva scheleriana y orteguiana sobre el amor nos puede permitir enlazar la actividad ética con la actividad sentimental, tanto del alumnado como del profesorado. En términos más específicos, los fundamentos filosóficos tratados en el apartado anterior permiten comprender que cada vez que el alumnado pone en práctica valores éticos para con las demás personas, sobre todo en el contexto de juego motor y/o deportivo, está vivenciando el amor hacia sus pares y/o hacia su profesorado. Cada vez que el alumnado, y el profesorado, escogen por medio de un acto intencional conservar la dignidad humana (Habermas, 2010; Ruiz, 2007) de sus compañeros, y alumnos, han experimentado el sentimiento de amor. Sentimiento que, por cierto, puede ser intuido por medio de las emociones que acompañan todos esos actos intencionales. Todas las veces que se promueve, y se practica, el respeto a los derechos humanos en las clases de EF, se están desarrollando actos amorosos que contribuyen en nuestro progreso humano. En resumen, cada vez que se ponen en práctica los valores éticos como la solidaridad, la responsabilidad, el perdón, la humildad, la tolerancia y el respeto, se está desarrollando una EF basada en el amor. En este sentido, sin ánimo de menospreciar el esfuerzo de otros especialistas en EF, se podría desarrollar un modelo de enseñanza de educación ética en EF mucho más amplio que el de Hellison (1978, 1985, 1995), ya que se utilizaría un concepto que incluye todos los valores éticos positivos y no solamente la responsabilidad persona y social. Dicho modelo podría llamarse, entre muchas otras denominaciones, educación basada en el amor en EF. Un modelo de este tipo podría contribuir a prevenir y abordar la violencia (simbólica, física, psicológica, sistémica) y el acoso (Bullying) escolar en EF, ya que es una problemática que ha sido reconocida en diversas investigaciones científicas en dicho contexto pedagógico (BascónSeda \& Ramírez-Macías, 2020; Butler, Burns \& Robson, 2020; Jiménez-Barbero, Jiménez-Loaisa, González-Cutre, BeltránCarrillo, Llor-Zaragoza \& Ruiz-Hernández, 2020; MartínezBaena \& Faus-Boscá, 2018; Monforte \& Úbeda-Colomer, 2019; Moura y Lovisolo, 2017). Precisamente, Martínez-Baena \& Faus-Boscá (2018), señalan que dichos fenómenos, dañinos a nivel general para el alumnado, pueden ser enfrentados con el aprendizaje de virtudes positivas durante la clase de EF.

Otra de las aplicaciones que pueden tener los fundamentos filosóficos del amor tratados en este ensayo, es la relación del alumnado, y del profesorado, en torno al sentimiento con la asignatura. En algunas investigaciones empíricas, coherentemente con estas perspectivas filosóficas, se ha demostrado que la vocación hacia la EF está asociada a un sentimiento que los estudiantes, y profesionales, en algunos casos han denominado explícitamente amor y en otras casos se aprecia de forma implícita por medio de los deseos vocacionales (Mujica \& Orellana, 2016, 2018, 2019). Evidentemente, ese profundo sentimiento pudo haber sido percibido por la vía emocional, es decir, por ejemplo, por la alegría o el entusiasmo que produce dicha asignatura pedagógica en las personas. Otro ejemplo, por el deseo de que la EF sea tratada con la buena consideración que se merece y que muchas personas puedan aprovecharla. Cabe mencionar, que amar la EF en los tiempos actuales en los que el medio ambiente se ha visto deteriorado por los excesos humanos es un sentimiento muy deseable, ya que dicha asignatura se encuentra directamente relacionada con el cuidado de la naturaleza. Cuidado que, por cierto, comienza con el cuidado de nuestro propio cuerpo y continúa con nuestros hábitos de vida activa y saludable. En otras palabras, ¿cómo podremos cuidar el planeta si no somos capaces de cuidar de nuestra propia corporalidad? Ojalá que la sociedad tome conciencia de la importancia de la EF y logre amarla más, o sea, la valore positivamente y trabaje para conservarla adecuadamente en la sociedad.

\section{Conclusiones y aplicaciones Prácticas}

Con relación al primer objetivo de este ensayo, de analizar el significado de amor que proponen dos filósofos asociados a la escuela fenomenológica, se concluye que desde su perspectiva amar es considerado un sentimiento objetivo de corte espiritual, o metafísico, y, a su vez, un acto intencional dirigido a lo valorado positivamente. Dicho sentimiento sería la base de todos los buenos sentimientos, deseos y pensamientos orientados a conservar adecuadamente lo amado. Sin embargo, también se reconoce que dichos significados, desde otras perspectivas, pueden ser ampliados o cuestionados. Por ello, es fundamental aplicarlos con prudencia y pensamiento crítico. Asimismo, contrastarlos con otros estudios filosóficos y científicos sobre el tema.

De acuerdo al segundo objetivo de este ensayo, asociar el amor a la formación ética en la educación física escolar, se concluye que es un acto muy deseable que podría contribuir a los siguientes aspectos: a) respeto de la dignidad humana y de los derechos humanos; b) solidaridad entre pares y con el profesorado; c) prevención de la violencia y acoso escolar; d) humildad para reconocer los propios defectos y virtudes a nivel ético-moral, así como las virtudes de los compañeros y del profesorado; e) tolerancia de las personas que tienen una cultura, un pensamiento y un actuar divergente al propio; f) perdonar a los pares, como al profesorado, cuando cometen errores que dañen la propia autoestima o autoconcepto. Por cierto, todo lo anterior en el marco de la legislación vigente de las diferentes naciones que incluyen en su sistema escolar la asignatura de EF.

A partir del potencial que podrían tener los fundamentos filosóficos del amor scheleriano y orteguiano, una de las aplicaciones con amplio alcance sería la de propiciar modelos pedagógicos basados en el amor, es decir, basados en todos los valores éticos positivos.

\section{Futuras líneas de investigación}

Se identifican las siguientes cuatro líneas de investigación en torno al amor en la EF escolar: a) indagar la relación de las emociones del alumnado, y del profesorado, en la EF y su relación con los valores éticos positivos; b) analizar los deseos en el alumnado, y el profesorado, para con la asignatura de EF y su relación con el valor que cada sujeto le otorga a dicha disciplina educativa; c) valorar los significados del profesorado de EF sobre el sentimiento del amor y contrastarlos con los significados filosóficos propuestos en este 
ensayo; y d) relacionar los sentimientos, deseos y pensamientos que inspiraron a los estudiantes de magisterio en $\mathrm{EF}$, con el valor que cada sujeto le otorga a dicha disciplina pedagógica.

\section{Referencias}

Alves, V., Barea, R., Werneck, V., Grzibowski, S., Rodrigues, D., \& Silva, L. (2018). Ethical care of the other: Edith Stein and Max Scheler's contributions. Escola Anna N e ry, 22(2), 1-6. doi: 10.1590/2177-9465-EAN-2017-0382

Alonso, J. (2017). Las emociones. La base neurológica del comportamiento. Barcelona: RBA.

Alonso, J., Marín, M., Yuste, J.L., Lavega, P., \& Gea, G. (2019). Conciencia emocional en situaciones motrices cooperativas lúdicas y expresivas en Bachillerato: perspectiva de género. Educatio Siglo XXI, 37(1), 195-212. doi: $10.6018 / \mathrm{j} / 363461$

Amilburu, G. (2014). Filosofía y actitud filosófica: sus aportaciones a la educación. Revista Española de Pedagogía, 72(258), 231-247.

Bascón-Seda, A., \& Ramírez-Macías, G. (2020). Víctimas de bullying: aportaciones para identificar casos de acoso en Educación Física dentro de la Educación Secundaria Obligatoria. Cultura, Ciencia y Deporte, 15(43), 109-119. doi: 10.12800/ccd.v15i43.1404

Butler, J., Burns, D. P., \& Robson, C. (2020). Dodgeball: Inadvertently teaching oppression in physical and health education. European Physical Education Review. doi: 10.1177/1356336X20915936

Cagigal, J. M. (1975). El deporte en la sociedad actual. Madrid: Prensa Española.

Cagigal, J. M. (1981a). ¡Oh deporte!, anatomía de un gigante. Valladolid: Miñón.

Cagigal, J. M. (1981b). En torno a la educación por el movimiento. Apunts. Medicina del L'esport, 18(72), 203-213.

Cagigal, J. M. (1984). ¿La Educación Física ciencia? Educación Física y Deporte, 6(2-3), 49-58. Recuperado de: https://revistas.udea.edu.co/index.php/ educacionfisicaydeporte/article/view/4716

Cagigal, J. M. (1986). En torno a la educación por el movimiento. Apunte antropofilosófico. Apunts. Educació Física I Esports, 6, 11-22.

Cagigal, J. M. (1996). Obras Selectas. Volumen III. Madrid: Comité Olímpico Español.

Castejón, F. J. (2005). Una aproximación a la utilización del deporte. El proceso de enseñanza aprendizaje. Lecturas: Educación Física y Deportes, 80, 1-1.

Castejón, F. J. (2010). Deporte como concepto y aplicación. En F. J. Castejón (Ed.), Deporte y Enseñanza Comprensiva (pp. 11-34). Sevilla: Wanceulen.

Castro, M. (2012). Educación Física, una mirada desde la filosofía. Motricidad y Persona, 10, 45-50.

Chaparro, M. (2014). Ordo amoris como determinante del amor y odio en Max Scheler. Veritas, 31, 51-71. Recuperado de: https://scielo.conicyt.cl/pdf/veritas/n31/art03.pdf

Devís, J. (1996). Educación Física, deporte y currículum. Investigación y desarrollo curricular. Madrid: Visor.

Fernández-García, L., \& Fernández-Río, J. (2019). Proyecto Wonderwall: identificación y manejo de emociones en la
Educación Física de Educación Primaria. Retos. Nuevas Tendencias en Educación Física, Deportes y Recreación, 35, 381-386.

Ferrater, J. (1998). La filosofía actual (12ª ed.). Madrid: Alianza.

Ferrater, J. (1994). Diccionario de grandes filósofos 1 (A-H). Madrid: Alianza.

Ferrater, J. (1995). Diccionario de grandes filósofos 2 (I-Z). Madrid: Alianza.

Fuster, D. (2019). Investigación cualitativa: método fenomenológico hermenéutico. Propósitos y Representaciones, 7(1), 201-221. doi: 10.20511/pyr2019.v7n1.267

García, L. M., \& Gutiérrez, D. (2017). Aprendiendo a enseñar deporte. Modelos de enseñanza comprensiva y educación deportiva ( $2^{\mathrm{a}}$ ed.). Barcelona: Inde.

Habermas, J. (2010). El concepto de dignidad humana y la utopía realista de los derechos humanos. Diánoia, 55(64), 3-25. Recuperado de: https://ialnet.unirioja.es/servlet/ articulo?codigo $=3344131$

Hellison, D. (1978). Beyond ball and bats: Alienated (and other) youth in the gym. Washington, DC.: American Alliance for Health, Physical Education, Recreation and Dance.

Hellison, D. (1985). Goals and strategies for physical education. Champaign, IL: Human Kinetics.

Hellison, D. (1995). Teaching responsibility through physical activity. Champaign, IL: Human Kinetics.

Jiménez-Barbero, J., Jiménez-Loaisa, A., González-Cutre, D., Beltrán-Carrillo, V., Llor-Zaragoza, L., \& Ruiz-Hernández, J. (2020). Physical Education and School bullying: a systematic review. Physical Education and Sport Pedagogy, 25(1), 79-100. doi: 10.108017408989.2019. 1688775

Kierkegaard, S. (2006). Las obras del amor. Meditaciones cristianas en forma de discursos. Salamanca: Sígueme.

Kirk, D. (2010). Physical Education Futures. New York: Routledge.

Kirk, D. (2017). Teaching Games in Physical Education. Towards a pedagogical model. Revista Portuguesa de Ciências do Desporto, Supl. 1, 17-26. doi: 10.5628/ rpcd.17.s1a.17

Lawson, H. (2009). Paradigms, exemplars and social change. Sport, Education and Society, 14(1), 97-119. doi: 10.1080/13573320802615247

Lawson, H. (2018). Physical Education in the industrial age school. En H. Lawson (Ed.), Redesigning Physical Education: An equity agenda in which every child matters (pp. 23-40). New York: Routledge.

Lawson, H. (2019). The Physical Education System as a Consequential Social Determinant. Quest, 72(1), 7284. doi:10.1080/00336297.2019.1627224

Lleixà, T. (2016). ¿Qué es realmente esto de las competencias? En T. Lleixà y E. Sebastiani (Eds.), Competencias clave y Educación Física (pp. 19-30). Barcelona: Inde.

Mari, G. (2014). La aportación del concepto de $<<$ persona $>>$ a la educación intercultural. Revista Española de Pedagogía, 72(258), 299-313.

Martínez-Baena, A., \& Faus-Boscá, J. (2018). Acoso escolar y Educación Física: una revisión sistemática. Retos. Nuevas Tendencias en Educación Física, Deportes y 
Recreación, 34, 412-419. Recuperado de: https:// recyt.fecyt.es/index.php/retos/article/view/59527/39906

Monforte, J., \& Úbeda-Colomer, J. (2019). 'Como una chica': un estudio provocativo sobre estereotipos de género en educación física. Retos. Nuevas Tendencias en Educación Física, Deportes y Recreación, 36, 74-79. Recuperado de: https://recyt.fecyt.es/index.php/retos/article/ view/68598/42123

Moura, D. y Lovisolo, H. (2017). Ritual of violence in Physical Education classes. Journal of Physical Education, 28, 1-11. doi: 10.4025/jphyseduc.v28i1.2822

Mujica, F. (2019). El dualismo cuerpo y alma en la Educación Física: análisis de las ideas de José María Cagigal. EmásF. Revista Digital de Educación Física, 60, 116-126. Recuperado de: https://dialnet.unirioja.es/servlet/ articulo? codigo $=7063110$

Mujica, F. (2020a). El término Educación Física en la posmodernidad: contribución de algunas perspectivas fenomenológicas. Retos. Nuevas Tendencias en Educación Física, Deportes y Recreación, 38, 795-801. Recuperado de: https://recyt.fecyt.es/index.php/retos/article/ view/73011/49514

Mujica, F. (2020b). Fundamentos para una educación física postcartesiana: análisis crítico a la ciencia de la motricidad humana. EmásF. Revista Digital de Educación Física, 63, 104-115.

Mujica, F., \& Inostroza, C. (2020). Políticas educativas, evaluaciones estandarizadas y formación escolar integral en Chile: análisis de la desvalorización a diferentes m a te rias del currículo. Dilemas Contemporáneos: Educación, Política y Valores, 7(2), 1-20.

Mujica, F., \& Orellana, N. (2020). Apreciaciones del profesorado chileno sobre una educación basada en el amor: estudio de casos. Revista Humanidades, 10(1), 1-16. doi: 10.15517/h.v10i1.39215

Mujica, F., \& Orellana, N. (2016). Construcción de la vocación en estudiantes de Pedagogía en Educación Física: un componente subjetivo de la formación profesional. Revista de Educación Física, 34(3), 1-7.

Mujica, F., \& Orellana, N. (2018). Autopercepción de la vocación en docentes de educación física escolar en Chile. CPU-e. Revista de Investigación Educativa, 27, 202-229.

Mujica, F., \& Orellana, N. (2019). Deseos vocacionales que incentivan a la formación docente en Educación Física. Revista Educación, 43(1), 1-12. doi: 10.15517/ revedu.v43i1.30013

Nussbaum, M. (2010). Sin fines de lucro. Por qué la democracia necesita de las humanidades. Madrid: Katz.

Nussbaum, M. (2016). Educación para el lucro, educación para la libertad. Nomadas, 44, 13-25. doi: 10.30578/ nomadas.n44a1

Oña,A. (2002). La ciencia en la actividad física: viejos y nuevos problemas. European Journal of Human Movement, 9,9-42.

Ortega y Gasset, J. (2018). Estudios sobre el amor (17 $7^{\mathrm{a}} \mathrm{ed}$.). Madrid: Edaf.

Perrino, M., \& Vicente, M. (2018). Olimpismo en la revista Citius, Altius, Fortius (1959-1976): los inicios de la crítica al Movimiento Olímpico en España. Retos Nuevas Tendencias en Educación Física, Deportes y Recreación,
34, 177-182.

Rodríguez, L. (2012). La esencia y las formas del amor según Max Scheler. Anuario Filosófico, 45(1), 69-96. Recuperado de: https://dadun.unav.edu/bitstream/10171/22897/ 2/04.pdf

Rojas, E. (1989). El laberinto de la afectividad. Madrid: Espasa-Calpe.

Rojas, E. (1993). Una teoría de la felicidad (16 a ed.). Madrid: Dossat.

Rosas, O. (2011). La estructura disposicional de los sentimientos. Ideas y Valores, 145, 5-31.

Ruiz, V. (2007). Derechos humanos, universales. En-claves del pensamiento, 1(1), 155-166.

Scheler, M. (1966). La esencia de la filosofía y la condición moral del conocer filosófico ( $3^{\mathrm{a}}$ ed.). Buenos Aires: Nova.

Scheler, M. (2001). Ética. Nuevo ensayo de fundamentación de un personalismo ético( $1^{\mathrm{a}} \mathrm{ed}$.). Madrid: Caparrós.

Scheler, M. (2005). Esencia y formas de la simpatía. Sígueme: Salamanca.

Scheler, M. (2008). Ordo amoris. Madrid: Caparrós.

Scheler, M. (2010). Amor y conocimiento. Y otros escritos. Madrid: Palabra.

Siedentop, D. (2002). Content knowledge for physical education. Journal of Teaching in Physical Education ,21,368-377. doi: 10.1123/jtpe.21.4.368

Velázquez, R., \& Hernández, J. L. (2010). La educación física: situación actual y calidad de enseñanza. En R. Velázquez y J. L. Hernández (Eds.), La educación física a estudio. El profesorado, el alumnado y los procesos de enseñanza (pp. 21-36). Barcelona: Graó.

Vendrell, Í. (2015). The emotions in early phenomenology. Studia Phaenomenologica, 15, 349-374. doi: 10.5840/ studphaen20151518

Vlieghe, J., \& Zamojski, P. (2019). Out of love for some-thing: an ontological exploration of the roots of teaching with Arendt, Badiou and Scheler. Journal of Philosophy of Education, 53(3), 518-530. doi: 10.1111/1467-9752.12375

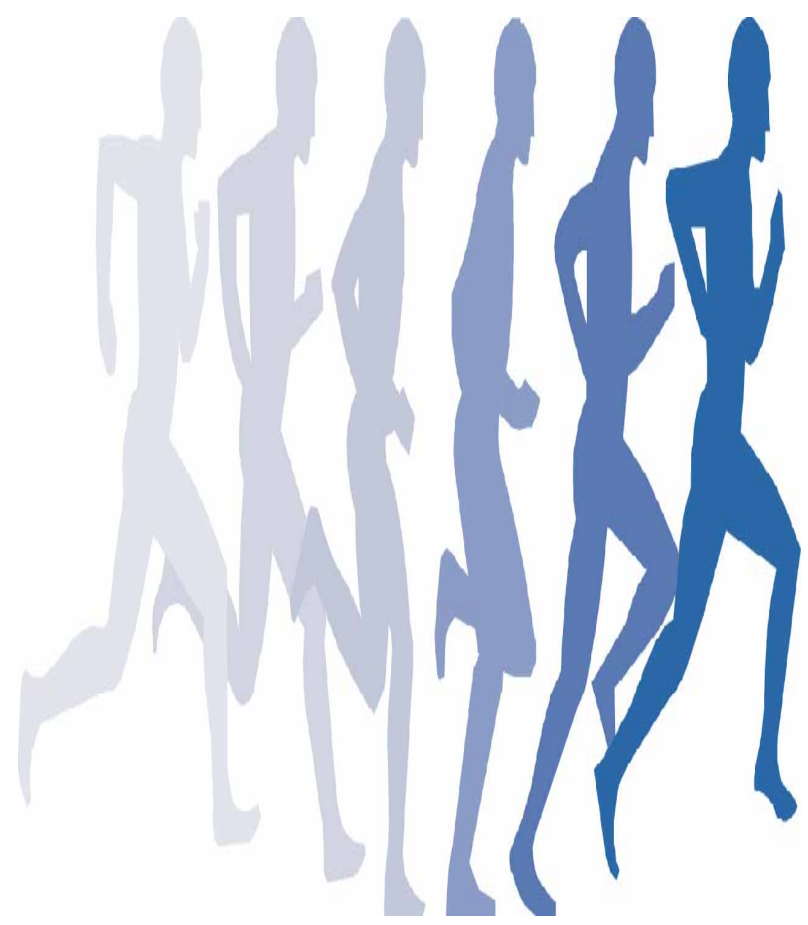

